\title{
¿Periodismo móvil o periodismo en la era de los móviles?
}

Mobile journalism or journalism in the mobile age?

Oscar Westlund - Oslo Metropolitan University, Volda University College, University of Gothenburg - oscar.westlund@oslomet.no

\section{Resumen}

La cantidad de formas en las que usamos y confiamos en nuestros dispositivos móviles hoy en día es fascinante. No existe una gran reflexión sobre la dependencia tecnológica en los dispositivos móviles y la comunicación en la vida diaria. Esto es sorprendentemente diferente a lo que sucedía en los diez años previos a la llegada de los smartphones contemporáneos con pantallas táctiles. El acceso a las noticias es más sencillo que nunca, incluso con la abundancia de noticias gratis difundidas a través de plataformas digitales ubicuas y dispositivos móviles. La conexión entre los dispositivos móviles y la cobertura informativa se ha convertido en uno de los avances más importantes del periodismo, aunque la investigación sobre esta materia es todavía escasa. Empezando por un análisis de los orígenes y el papel actual del periodismo móvil, este artículo concluye abordando algunas cuestiones específicas relacionadas con la investigación sobre la producción, distribución y consumo de las noticias móviles. Existe la necesidad de desarrollar una mejor comprensión intercultural e interdisciplinar de la conexión entre los dispositivos móviles y el periodismo a través del uso de diversos conjuntos de métodos.

\section{Palabras clave}

Comunicación móvil, periodismo móvil, dispositivos móviles, smartphones, producción informativa.

Sumario

1. Introducción. 2. Mirada retrospectiva de la emergencia del periodismo y noticias para dispositivos móviles. 3. ¿Periodismo móvil? 4. Hacia una agenda de investigación. 5. Bibliografía. 


\section{Introducción}

En el momento en el que escribo esto (primavera de 2018), los smartphones con pantalla táctil ya llevan una década en el mercado. El periodismo, los diferentes tipos de medios informativos, y los flujos diarios de noticias, mucho más tiempo. Hasta la fecha, muchos reportajes dieron voz a la combinación de ambos. Los medios informativos y los periodistas se han vuelto dependientes de la tecnología (Lewis y Westlund, 2016), incluidos, entre otros, cómo se utilizan los dispositivos móviles en la producción y distribución de noticias (Westlund, 2011, 2013). En la última década, el consumo de noticias móviles ha cobrado importancia entre los ciudadanos de casi todo el mundo (Newman; Fletcher; Kalogeropoulos; Levy y Kleis Nielsen, 2017; Westlund, 2014), se está usando tanto en la vida diaria (Dimmick, Feaster y Ramirez, 2011) y en muchos espacios desde la mañana hasta la noche (Van Damme, Courtois, Verbrugge y De Marez, 2015; Westlund, 2015). Algunas noticias móviles han experimentado un efecto de desplazamiento y otras un efecto complementario en otras rutinas de consumo mediático (Thorson, Shoenberger, Karaliova, Kim y Fidler, 2015; Westlund y Färdigh, 2015). En términos generales, el usuario digital contemporáneo es una criatura compleja (Picone, 2016), que se involucra en una gran variedad de prácticas digitales de consumo de noticias (Costera Meijer y Groot Kormelink, 2015), que incluyen cómo los usuarios distribuyen noticias con dispositivos móviles (Villi y Matikainen, 2015).

Con los cambios en el consumo de noticias, surgen muchas preguntas, como las preguntas normativas sobre si las noticias móviles, en su mayor parte, son "buenas" o malas" para la sociedad y sus ciudadanos. Depende. En algunas partes del mundo, el dispositivo móvil ha brindado a las personas la primera puerta de entrada a la World Wide Web, así como un sinfín de fuentes de noticias de todo el mundo. Esto es claramente mucho mejor que no tener acceso. Estudios de Estados Unidos, por ejemplo, llegaron a la conclusión de que el teléfono móvil desempeña un papel crucial en la forma en que las minorías éticas acceden a las noticias (Richardson, 2016), y en cómo se involucran en la vida política (Martin, 2015). Sin embargo, algunos han defendido que el consumo de noticias a través de dispositivos móviles ha ocasionado que las personas dediquen un tiempo limitado a las noticias, solo echándoles un ojo (Molyneux, 2017), en lugar de desarrollar un aprendizaje más profundo sobre las noticias y los asuntos públicos. Sin embargo, hay que tener en cuenta que el consumo de noticias móviles es complejo y multifacético, y los patrones de consumo rápido en espacios de tiempo dedicados a otras cosas mientras estamos en movimiento, puede complementarse con lecturas más largas en el sofá o la cama por la noche. Los consumidores de noticias en el móvil tienden a usar sus dispositivos móviles en múltiples ocasiones a lo largo del día, por lo que el tiempo que dedican a las noticiases elevado (Westlund, 2016).

En muchas partes del mundo, los ciudadanos, a la hora de consumir noticias, están migrando, de forma más o menos activa, de las plataformas mediáticas tradicionales, en las que los periodistas y editores presentan su edición seleccionada de noticias, a los dispositivos móviles. La oferta de noticias en una aplicación o en una web móvil varía sustancialmente, dependiendo de las prácticas de producción de noticias (Westlund, 2011), los cambios a medida que pasa el tiempo en el contenido de las noticias seleccionado para su inclusión (Nel y Westlund, 2012), y también de los países (López García, Westlund y Silva, 2015). En general, la combinación de periodismo y noticias con dispositivos móviles supone una alteración en la forma en la que los medios de comunicación y los periodistas producen y distribuyen noticias, y en cómo la gente se involucra en el consumo de noticias en el móvil. Muchas cosas han ocurrido en las primeras dos décadas del siglo XXI.

\section{Mirada retrospectiva de la emergencia del periodismo y noticias para dispositivos móviles}

Mi primera experiencia de acceso a noticias con un teléfono móvil fue a principios de este milenio, cuando usé mi Nokia para acceder a Vodafone Live, un jardín vallado de unidades móviles inspirado por la NTT DoCoMo en Japón. Lo hice en parte por curiosidad para ver si era posible, en parte porque estaba de vacaciones y porque no tenía otra forma de acceder a las noticias. Tuve que esperar, y esperar, y esperar un poquito más, para que la noticia se cargara en la pequeña pantalla de mi teléfono. El jardín vallado solo daba acceso a una selección limitada de noticias y fuentes, según el acuerdo entre el operador de telecomunicaciones y los editores. No había dispositivos táctiles en el mercado, los costes por utilizar datos móviles eran caros, y los tiempos de carga eran lentos. En ese momento, la interfaz para acceder a noticias en el móvil no era fácil de usar, y los medios de comunicación no producían noticias para teléfonos móviles.

Ahora, muchos teléfonos móviles son ultraportátiles e incorporan funcionalidades como reproductor de música, radio, mensajería e internet. Lo que antes se conocía como teléfono móvil es ahora una poderosa tecnología de información y comunicación (TIC) con internet, que permite a sus usuarios acceder e interactuar con diversos tipos de audio, vídeo, gráficos, texto y animación. Ha experimentado tales avances y transformaciones que ya no tiene sentido hablar de teléfono, sino de dispositivo móvil. En este momento se están utilizando diferentes tipos de dispositivos móviles, desde pequeños dispositivos ponibles como relojes inteligentes -por ejemplo, el Samsung Gear- que se pueden usar para notificaciones de noticias, auriculares portátiles con asistentes virtuales (como el Bose Quiet Comfort II), que lee en voz alta podcasts de noticias, o teléfonos inteligentes (como el iPhone de Apple), y tabletas (como el Apple iPad), que sirven como puertas de entrada a todo tipo de periodismo de noticias. Todos ellos se consideran tecnologías personales y portátiles con conectividad a internet (entre los cuales algunos actúan como extensión de otro dispositivo conectado). En aras de la claridad, el concepto de dispositivo móvil se referirá aquí a lo que de otra manera y popularmente se conoce como teléfonos inteligentes. Este es el dispositivo móvil personal y portátil más difundido. Los avances tecnológicos en hardware y software han derivado en teléfonos que ofrecen funcionalidades y cualidades similares a las de un ordenador, muchas de las cuales están vinculadas a su acceso ubicuo a internet. La pantalla táctil, las aplicaciones personalizadas y muchas otras propiedades específicas para teléfonos móviles contribuyen a la construcción de una interfaz móvil única. Algunas de estas propiedades están en primer plano y son muy evidentes para los usuarios de los móviles, como los cambios en el tamaño, forma y resolución de la pantalla, mientras que puede decirse que otras propiedades como los servicios de posicionamientos que "saben" dónde está el usuario en relación con sus conocidos, monumentos históricos y publicistas, funcionan en segundo plano para ofrecer al usuario (y anunciante) una entrega personalizada. La interfaz de usuario está inexorablemente conectada a las posibilidades y limitaciones que rodean el uso de móviles, y por tanto influirá en la experiencia de consumo de noticias móviles. Una aplicación móvil de noticias personalizadas (llamada aplicación nativa) para iPhone o Android, respectivamente, puede hacer la que experiencia de usuario varíe sustancialmente. Además, la experiencia de usuario con el mismo modelo o un dispositivo móvil específico (como el iPhone X), puede variar sustancialmente si el usuario accede a una aplicación nativa, visita una página web, un sitio móvil o un sitio diseñado para parecerse a una aplicación (por ejemplo, aplicaciones web progresivas). 
En conjunto, estos desarrollos tecnológicos han facilitado la producción, distribución y consumo de noticias en el móvil, que se han convertido en algo habitual para la sociedad, pero todavía comprenden un área de investigación emergente y relativamente inmadura en la intersección entre los estudios de periodismo, por un lado, y los medios móviles y la comunicación, por otro. Han pasado quince años desde que empecé a investigar la relación entre los móviles, las noticias y el periodismo, y la producción, la distribución y el consumo. Por aquel entonces, los medios de comunicación empezaban a experimentar con tecnologías novedosas como SMS y sitios de noticias básicos, y casi ninguna persona recurría a su teléfono móvil para acceder a las noticias. Con el tiempo he llevado a cabo varios estudios sobre la producción y distribución de noticias para dispositivos móviles (Westlund, 2012; Westlund, 2011), mapas de contenido y funcionalidades de noticias móviles (López García et al., 2015; Nel y Westlund, 2012). ), he realizado estudios que vinculan las preferencias y los patrones de consumo de noticias móviles (Westlund, 2007, 2010), he investigado la transformación de los patrones de consumo de noticias en el tiempo y entre grupos (Westlund y Bjur, 2014; Westlund, 2008), he estudiado desplazamientos y efectos complementarios en otras formas de consumo de noticias (Westlund y Färdigh, 2015; Westlund y Weibull, 2013). Me han invitado en numerosas ocasiones a escribir artículos de investigación centrados en noticias móviles o periodismo móvil (Westlund, 2013; Westlund, 2014, 2016a), incluida la publicación reciente de entradas de artículos de enciclopedia sobre estos temas (Westlund, 2018; Westlund y Quinn, 2018). Con el tiempo, ha habido un crecimiento constante en la investigación de móviles en los estudios sobre noticias y el periodismo, pero, sorprendentemente, todavía hay poca producción científica en ese sentido. Algunas preguntas clave todavía no tienen respuesta. Entre ellas, si existe el llamado "periodismo móvil", o si se trata simplemente de periodismo en la era de los medios móviles. Ahora hablamos de esto.

\section{3. ¿Periodismo móvil?}

Uno podría cuestionar si existe algo así como el periodismo móvil en los casos en que los medios de comunicación aplican procesos en los que el mismo contenido de noticias que se produce, se publica y distribuye a través de varias plataformas digitales y móviles a la vez. Es decir, cuando las noticias producidas son independientes de la plataforma, y donde se hace poco para distribuirlas a los consumidores de noticias en el móvil de una forma distinta. Por lo general, el periodismo digital contemporáneo integra el trabajo periodístico cross-media, donde se producen y publican noticias simultáneamente para varias plataformas diferentes (tanto propietarias como no propietarias), y donde las noticias deberían poder desplazarse de una plataforma a otra. En este contexto, por lo general, tiene más sentido pensar en el periodismo en una era de medios móviles que en una forma distinta de periodismo móvil. Sin embargo, algunos factores y prácticas apuntan en la dirección del periodismo móvil, en el sentido que las herramientas, los sistemas y las plataformas móviles desempeñan un papel importante en la producción y distribución de noticias.

En primer lugar, las prácticas para el periodismo móvil varían entre diferentes medios de comunicación y en el tiempo. Algunos medios tradicionales experimentaron con la publicación de noticias para buscapersonas, asistentes personales digitales (PDA) y los teléfonos con funciones de la época. La elaboración manual y la publicación de notificaciones de alerta de noticias, enviadas por SMS o MMS a suscriptores con teléfonos de funciones estándar, empezaban a ponerse en práctica. A principios de los años 2000, los editores de noticias de todo el mundo ofrecían alertas de las últimas noticias, incluyendo Asia, Europa y Sudamérica. Las compañías de noticias como The Guardian y Stampen han contratado periodistas dedicados a personalizar el contenido de noticias para aplicaciones móviles y páginas (ver, por ejemplo, Westlund, 2013). Un segundo factor implica que la personalización de noticias en el móvil todavía puede llevarse a cabo por medio de la entrega. Como se comentó anteriormente, la personalización de contenido en el móvil es una opción de dispositivos móviles importante y que se usa con frecuencia. Las aplicaciones de noticias propias de medios de comunicación tradicionales, o aplicaciones agregadoras de noticias no proprietarias como Apple News, Facebook Instant Articles, Flipboard, Google News y Omni publican noticias basadas en preferencias y comportamientos de un gran grupo de editores de noticias. Además, desde hace tiempo se ha contemplado que los dispositivos móviles, que se pueden usar en cualquier lugar, pueden vincular el contenido al espacio específico y al contexto en el que se usa el móvil. Los investigadores han explorado esto con conceptos tales como noticias locativas, periodismo locativo y periodismo espacial (ver más lecturas y referencias cruzadas).

En tercer lugar, incluso si las noticias no siempre se personalizan para su publicación en plataformas móviles, los dispositivos pueden desempeñar un papel esencial en la forma en que los periodistas producen las noticias. Desde hace tiempo, el periodismo depende de la tecnología para la producción y distribución de noticias. Los dispositivos móviles son cada vez más importantes para el periodismo, especialmente para los periodistas con múltiples habilidades que trabajan de forma autónoma. Las primeras iniciativas como iReport de la CNN y MoJo Kit (una colaboración entre Reuters y Nokia) comenzaron a allanar el camino para los llamados periodistas móviles (Mojos). Tanto la formación reglada y el aprendizaje experiencial e indirecto más informal han facilitado las prácticas de Mojo (Westlund, 2013; c.f. Westlund y Quinn, 2018). Los Mojos usan sus dispositivos móviles de forma activa en los procesos de producción de noticias: para comunicarse de diversas formas con sus fuentes de información, para escribir textos, hacer fotos, grabar y editar videos, y también para publicar su trabajo (Burum y Quinn, 2016). El surgimiento del periodismo móvil ha transformado los procesos de producción de noticias tanto a pie de calle como en las redacciones. Algunas investigaciones recientes de Hindustan Times en la India apuntan que los periodistas experimentan la práctica de MoJo tanto en la capacitación (enskilling) como en la descapacitación (unskilling) (Kumar y Mohamed Haneef, 2017).

Los periodistas contemporáneos pueden usar sus dispositivos móviles para comunicarse con las fuentes, con o sin cifrado, a través de una amplia gama de aplicaciones de chat y mensajería de fácil acceso. Hasta la fecha, los teléfonos móviles como el iPhone de Apple y la serie de Samsung Galaxy S permiten que los periodistas se comuniquen fácilmente con sus compañeros, fuentes de información y audiencia a través de la voz, SMS, correo electrónico y diferentes medios sociales. También pueden utilizar sus dispositivos móviles para prácticas periodísticas como blogs en vivo, así como para hacer, editar y publicar vídeos y foto (Westlund, 2013). En cuarto lugar, el periodismo móvil abarca una estrecha conexión al periodismo ciudadano, lo que permite capturar momentos por azar. Durante décadas, los ciudadanos se han nutrido de los procesos de producción de noticias a través de llamadas a los periodistas. Equipados con dispositivos móviles con cámara para fotos y vídeo, han podido producir con facilidad testimonios de acontecimientos noticiosos en el lugar de los hechos. A golpe de unos pocos clics, y a través de la conectividad a internet del dispositivo móvil, pueden enviar material de noticias a los medios de comunicación, publicar ellos mismos en páginas web, y lanzar contenido a redes sociales (ver, por ejemplo, Westlund, 2013). El uso combinado de dispositivos móviles y redes sociales como Twitter ha brindado a los ciudadanos los medios para producir, publicar y acceder a noticias relevantes para ellos. Richardson (2017) analizar el surgimiento y la prevalencia del testimonio negro a través del móvil en Estados Unidos, una forma de periodismo digital producido y anhelado por los grupos maginados de la sociedad. Su artículo destaca cuánto de importante puede ser el periodismo móvil impulsado por los ciudadanos para los distintos grupos sociales. 


\section{Hacia una agenda de investigación}

En suma, la producción científica hasta la fecha nos ha aportado reflexiones relevantes sobre la combinación constante de periodismo y medios móviles. Sin embargo, la literatura existente solo araña la superficie. Expresiones de moda como big data, medios sociales y fake news han llamado la atención de académicos de todo el mundo, lo que eclipsó el potencial de investigación sobre periodismo en la era de los medios móviles. Asimismo, los dispositivos móviles están inexorablemente conectados al desarrollo del big data, medios sociales y fake news. Es necesario desarrollar un mejor conocimiento transcultural y transversal del nexo entre dispositivos móviles y periodismo, con la ayuda de diferentes métodos. Por último, y quizás lo más importante, los estudios en el ámbito del periodismo deberían hacer más esfuerzos para abordar el papel (distintivo) de los móviles con enfoques más amplios para el estudio del periodismo contemporáneo.

\section{Bibliografía}

Burum, I. y Quinn, S. (2016). MOJO: the mobile journalism handbook: how to make broadcast videos with an iPhone or iPad. Focal Press, Taylor \& Francis.

Costera Meijer, I. y Groot Kormelink, T. (2015). Checking, Sharing, Clicking and Linking. Digital Journalism, 3(5), 664-679. https://doi.org/10.1080/21670811.2014.937149

Dimmick, J., Feaster, J. C. y Ramirez, A. (2011). The niches of interpersonal media: Relationships in time and space. New Media \& Society, 13(8), 1265-1282. https://doi.org/10.1177/1461444811403445

Kumar, A. y Mohamed Haneef, M. S. (2017). Is Mojo (En)De-Skilling?: Unfolding the practices of mobile journalism in an Indian newsroom. Journalism Practice, 2786(October), 1-19. https://doi.org/10.1080/17512786.2017.1389291

Lewis, S. y Westlund, O. (2016). Mapping the Human-Machine Divide in Journalism. En Witschge Tamara, A. Chris, D. David y H. Alfred (Eds.), Handbook of Digital Journalism. Londres: SAGE Publications.

López García, X., Westlund, O. y Silva, A. (2015). Prensa, smartphones y tablets. La industria de medios impresos se sube al periodismo móvil : Press, Smartphones and Tablets. Printed Media Industry Joins Mobile Journalism. Telos: Cuadernos de Comunicación e Innovación, 100, 128139. Recuperado de https://telos.fundaciontelefonica.com/url-direct/pdfgenerator?tipoContenido=articuloTelos\&idContenido $=2015030311040001$ \&idioma=es

Martin, J. A. (2015). Mobile news use and participation in elections: A bridge for the democratic divide? Mobile Media \& Communication, 3(2), 230249. https://doi.org/10.1177/2050157914550664

Molyneux, L. (2017). Mobile News Consumption. Digital Journalism, 811(June), 1-17. https://doi.org/10.1080/21670811.2017.1334567

Nel, F. y Westlund, O. (2012). The 4C'S of Mobile News: Channels, conversation, content and commerce. Journalism Practice, 5(6), 744-753. https://doi.org/10.1080/17512786.2012.667278

Newman, N., Fletcher, R., Kalogeropoulos, A., Levy, D. A. L. y Kleis Nielsen, R. (2017). The Reuters Institute's Digital News Report 2017. RISJ The Reuters Institute's Digital News Report 2017. Oxford.

Picone, I. (2016). Grasping the Digital News User. Digital Journalism, 4(1), 125-141. https://doi.org/10.1080/21670811.2015.1096616

Richardson, A. V. (2016). Bearing Witness While Black. Digital Journalism, 811(August), 1-26. https://doi.org/10.1080/21670811.2016.1193818

Richardson, A. V. (2017). Bearing Witness While Black. Digital Journalism, 5(6), 673-698. https://doi.org/10.1080/21670811.2016.1193818

Thorson, E., Shoenberger, H., Karaliova, T., Kim, E. y Fidler, R. (2015). News use of mobile media: A contingency model. Mobile Media \& Communication, 3(2), 160-178. https://doi.org/10.1177/2050157914557692

Van Damme, K., Courtois, C., Verbrugge, K. y De Marez, L. (2015). What's APPening to news? A mixed-method audience-centred study on mobile news consumption. Mobile Media \& Communication, 3(2), 196-213. https://doi.org/10.1177/2050157914557691

Villi, M. y Matikainen, J. (2015). Mobile UDC: Online media content distribution among Finnish mobile Internet users. Mobile Media \& Communication, 3(2), 214-229. https://doi.org/10.1177/2050157914552156

Westlund, O. (2007). The adoption of mobile media by young adults in Sweden. Mobile Media, 116-224. Recuperado de: http://biblioteca.esec.pt/cdi/ebooks/docs1/Adoption_Mobile_media.pdf

Westlund, O. (2008). From Mobile Phone to Mobile Device: News Consumption on the Go. Canadian Journal of Communication, 33, 443-463. https://doi.org/http://dx.doi.org/10.1108/17506200710779521

Westlund, O. (2010). New(s) functions for the mobile: a cross-cultural study. New Media \& Society, 12(1), 91-108.

https://doi.org/10.1177/1461444809355116 
Westlund, O. (2011). Cross-Media News work Sensemaking of the Mobile Media (R)evolution. University of Gothenburg, 1-367.

Westlund, O. (2012). Producer-centric versus participation-centric: On the shaping of mobile media. Northern Lights, 10(1). https://doi.org/10.1386/nl.10.107-1

Westlund, O. (2013). Mobile News. Digital Journalism, 1(1), 6-26. https://doi.org/10.1080/21670811.2012.740273

Westlund, O. (2013). Mobile news: A review and model of journalism in an age of mobile media. Digital Journalism, 1(1). https://doi.org/10.1080/21670811.2012.740273

Westlund, O. (2014). The Production and Consumption of News in an Age of Mobile Media. The Routledge Companion to Mobile Media, 145. https://doi.org/10.1080/21670811.2012.740273

Westlund, O. (2015). News consumption in an age of mobile media: Patterns, people, place, and participation. Mobile Media and Communication, 3(2). https://doi.org/10.1177/2050157914563369

Westlund, O. (2016a). Mobile News - The future of digital journalism. En B. Franklin \& S. Eldridge II (Eds.), The Routledge Companion to Digital Journalism Studies. Londres: Routledge.

Westlund, O. (2016b). News Consumption Across Media: Tracing the Revolutionary Uptake of Mobile News. En J. Linaa Jensen, M. Mortensen, \& J. Ormen (Eds.), News Across Media: Production, Distribution and Consumption (pp. 123-141). New York, New York, USA: Routledge.

Westlund, O. (2018). Mobile Journalism. En The International Encyclopedia of Journalism Studies. Wiley.

Westlund, O. y Bjur, J. (2014). Media Life of the Young. Young, 22(1). https://doi.org/10.1177/11103308813512934

Westlund, O. y Färdigh, M. A. (2015). Accessing the news in an age of mobile media: Tracing displacing and complementary effects of mobile news on newspapers and online news. Mobile Media and Communication, 3(1). https://doi.org/10.1177/2050157914549039

Westlund, O. y Quinn, S. (2018). Mobile journalism and MoJos. En Oxford Encyclopedia of Journalism Studies. Oxford University Press.

Westlund, O. y Weibull, L. (2013). Generation, life course and news media use in Sweden 1986-2011. Northern Lights, 11(1).

https://doi.org/10.1386/nl.11.147-1

\section{Cómo citar este artículo en bibliografías - How to cite this article in bibliographies / references:}

WESTLUND, O. (2018): ¿¿Periodismo móvil o periodismo en la era de los medios móviles?". En Revista de la Asociación Española de Investigación de la Comunicación, vol. 5, número 10, pp. 10-14. 